\title{
Effects of Domain Morphology on Kinetics of Fluid Phase Separation
}

\author{
Sutapa Roy and Subir K. Das* \\ Theoretical Sciences Unit, Jawaharlal Nehru Centre for Advanced Scientific Research, Jakkur P.O, Bangalore 560064, India
}

(Dated: June 22, 2021)

\begin{abstract}
Kinetics of phase separation in a three dimensional single-component Lennard-Jones fluid, that exhibits vapor-liquid transition, is studied via molecular dynamics simulations after quenching homogeneous systems, of different overall densities, inside the coexistence region. For densities close to the vapor branch of the coexistence curve, phase separation progresses via nucleation of liquid droplets and collisions among them. This is different from the evaporation-condensation mechanism proposed by Lifshitz and Slyozov, even though both lead to power-law growth of average domain size, as a function of time, with an exponent $\alpha=1 / 3$. Beyond a certain threshold value of the overall density, we observe elongated, percolating domain morphology which suddenly enhances the value of $\alpha$. These results are consistent with some existing theoretical expectations.
\end{abstract}

\section{INTRODUCTION}

When a homogeneous system is quenched inside the coexistence curve the system phase separates into particlerich and particle-poor domains. There has been longstanding interest in the kinetics of such processes 1 4]. Important example systems are solutions of multicomponent solids and fluids, vapor-liquid systems, etc. Depending upon the overall density $(\rho)$ or composition (in case of a mixture), the phase separation can be spontaneous or the onset can get delayed. For quenches very close to the coexistence curve, the system is less saturated and so waits for droplet nucleation [5-8] events which occur via rare long wavelength fluctuations. The range of density or composition for which this is true is referred to as the nucleation regime. Beyond this the kinetics of phase separation is termed as spinodal decomposition [3, 4] and the boundary between these two regimes is referred to as the spinodal line [4], a concept drawn from mean field theory and may be valid for long molecular systems, e.g., polymers. Nevertheless, there have been attempts to draw this line even for small molecular systems.

During the phase separation, transport mechanism plays crucial role [3] in the growth of average domain size $(\ell)$. Typically, $\ell$ exhibits power-law enhancement with time $(t)$ as 1 [ 3 ]

$$
\ell(t) \sim t^{\alpha}
$$

For diffusive transport, the growth exponent is $\alpha=1 / 3$, a value predicted by Lifshitz and Slyozov (LS) [9 11]. For phase separation in solid mixtures, at moderately high temperatures $(T)$, the LS value is the only exponent (at worst with minor corrections at small length limit) irrespective of whether the system is in nucleation or spinodal regime [10 12]. The situation in fluids, as described below, however, is not as simple.

Because of influence of hydrodynamics, the process, of course, is expected to be significantly faster in fluids. The hydrodynamic effects can show up either as an enhancement in the value of $\alpha$ or in the amplitude of growth. This fact is expected to be strongly dependent upon domain pattern.

For disconnected droplet morphology, close to the coexistence curve, Binder and Stauffer [13, 14] pointed out that the growth should occur via sticking of droplets following inelastic collisions. In that case, if the droplets exhibit random diffusive motion, the droplet density $(n)$ should decrease with time as [15]

$$
\frac{d n}{d t}=C_{1} D_{\ell} \ell n^{2}
$$

where $C_{1}$ is a constant and $D_{\ell}$ is the droplet diffusivity. Treating $D_{\ell} \ell$ as a constant, in accordance with the Stokes-Einstein-Sutherland (SES) relation [16], and using $n \propto \frac{1}{\ell^{3}}$, one obtains

$$
\frac{d \ell}{d t} \propto \frac{1}{\ell^{2}}
$$

The solution of Eq. (3) provides $\alpha=1 / 3$. Tanaka 17 19 argued that in high droplet density limit, motion of the droplets will not be random due to inter-droplet interaction. This was recently confirmed by us [20, 21]. Nevertheless, in this case also $\alpha$ should be $1 / 3$ but a difference will occur in the amplitude of growth.

Note that the collision mechanism will be prominent once there are well formed droplets in the system. At early enough time, it is possible that the domains (with density or composition inside them reasonably away from the equilibrium coexistence value) are interconnected to give rise to a different mechanism and exponent. This we discuss next.

At high overall density or concentration of the minority component in a binary mixture, the interconnected morphology, mentioned above, remains there for all time. In this case, at the beginning, the simple particle diffusion mechanism, as in solids, plays the dominant role. With increasing domain size, of course, hydrodynamics is expected to take over. As suggested by Siggia [15], hydrodynamic mechanism, in this case, helps fast material flow through the tube like elongated domains due to the pressure gradient created by interfacial tension $(\gamma)$ in the undulated tube geometry. Consideration of Poiseuille flow in such a geometrical and physical situation will provide

$$
\ell=C_{2} t
$$

where the constant of proportionality $C_{2}$ is a function of $\gamma$ and viscosity $(\eta)$. Eq. (4) is referred to as the viscous hydrodynamic growth law which at later time is expected to crossover to a slower growth with $\alpha=2 / 3$, referred to as the inertial hydrodynamic growth.

Eq. (4) and other growth laws in domain coarsening systems can be obtained from simple dimensional arguments as well. Success of such approach is inherent in the 
fact that the systems, under discussion, remain invariant under appropriate time and length rescalings 22].

Starting from the Navier-Stokes equation [16]

$$
\rho \frac{D}{D t} \vec{u}-\rho \eta \nabla^{2} \vec{u}=-\vec{\nabla} P
$$

with $D / D t=d / d t+(\vec{u} . \vec{\nabla}), \rho$ the mass density, $\eta$ the kinetic viscosity, $P$ the pressure and $u$ being the fluid velocity, from dimensional substitutions, Furukawa [22] arrived at the equation

$$
\frac{d \ell}{d t}+f \ell\left(\frac{d \ell}{d t}\right)^{2}=\frac{t_{0} \gamma}{\eta \xi \rho},
$$

which is a balance between frictions and force due to surface tension. In Eq.(6), $f$ is a friction coefficient and $t_{0}$ is a relaxation time related to the equilibrium correlation length $\xi$ that has the critical divergence

$$
\xi \sim \epsilon^{-\nu} ; \epsilon=\left|T-T_{c}\right|,
$$

$T_{c}$ being the critical temperature and $\nu$ a critical exponent. The quantities $\xi$ and $t_{0}$ are used to make $\ell$ and $t$ dimensionless in Eq. (6). From the decay of the dynamic structure factor [16], one can relate the time constant $t_{0}$ with $\xi$ as

$$
t_{0} \sim \frac{\xi^{2}}{D_{\xi}},
$$

$D_{\xi}$ being the diffusivity of clusters of the size of $\xi$. Again, using the SES relation [16], one can write

$$
\eta \sim \frac{1}{\xi D_{\xi}} .
$$

Furthermore, in space dimension $(d)$ three, $\gamma$ has the critical singularity [23]

$$
\gamma \sim \xi^{-2}
$$

All our results in this paper are from $d=3$. Combining Eqs. (8, 10), one obtains [22] the right hand side of Eq. (6) to be $\mathcal{O}(1)$. Note that in deriving Eq. (6), an important assumption made was that there exist unique time and length scales in the problem that give rise to unique velocity, i.e.,

$$
u=\frac{d \ell}{d t}
$$

In Eq. (6), Furukawa [22] identified the first and second terms on the left hand side as dissipative friction and inertial friction, respectively. Neglecting the inertial term, one obtains $\alpha=1$ and considering only the inertial term one gets $\alpha=2 / 3$. The lowering of the exponent from 1 to $2 / 3$ can be associated with turbulence in the system because of which break-up of well grown domains into smaller ones is possible. The turbulent character of the system at late time means that the Reynold's number is domain-size dependent. Physically, for big enough domains, viscosity may fail to hold the domains. Nevertheless, in the competition between growth and break-up, the system effectively continues to grow.

Our primary objective in this work is to test the above mentioned morphology dependence of the hydrodynamic growth mechanisms and thus the growth laws, via computer simulations. For the sake of convenience, we have chosen vapor-liquid system. Note that in a binary or multi-component fluid mixture, one needs to choose a high overall density to avoid interference between liquidliquid and vapor-liquid transitions. Further, for studies close to the coexistence curve, one needs very large systems to obtain reasonable droplet statistics. This fact, in case of a fluid mixture, can cause severe computational difficulty, since the overall density of the system must remain fixed.

Our observations in the vapor-liquid systems, that we have studied, confirm the above theoretical expectations for the morphology dependence of the growth exponents. We provide supportive results that the growth exponent $\alpha=1 / 3$, observed for low enough density, is due to a different mechanism than the LS mechanism. In addition, we present important results for various functions related to the pattern formation in coarsening systems.

The rest of the paper is organized as follows. In Section II we discuss the model and methods. Results are presented in Section III. Finally, the paper is summarized in Section IV.

\section{MODEL AND METHODOLOGIES}

In our model 24 system, particles, of equal size and mass $(m)$, at continuous positions $\vec{r}_{i}$ and $\vec{r}_{j}$ in a cubic box (unless otherwise mentioned) of linear dimension $L$ (in units of particle diameter), interact via

$$
U\left(r=\left|\vec{r}_{i}-\vec{r}_{j}\right|\right)=u(r)-u\left(r_{c}\right)-\left.\left(r-r_{c}\right) \frac{d u}{d r}\right|_{r=r_{c}}
$$

where

$$
u\left(r_{i j}\right)=4 \varepsilon\left[(\sigma / r)^{12}-(\sigma / r)^{6}\right]
$$

is the standard Lennard-Jones (LJ) potential with $\varepsilon$ and $\sigma$ being respectively the interaction strength and particle diameter. In this model, the cut-off radius $r_{c}$ was introduced for the sake of computational benefit. The last term in Eq. (12) ensures the continuity of both potential and force at $r_{c}=2.5 \sigma$. The critical temperature and critical density $\left(\rho_{c}\right)$ for the vapor-liquid transition, that this model exhibits, were estimated [25] to be $k_{B} T_{c} \simeq 0.9 \varepsilon$ ( $k_{B}$ being the Boltzmann constant) and $\rho_{c} \simeq 0.3$ (note that the density $\rho$ is defined as $\frac{N \sigma^{3}}{V}, N$ being the number of particles in the system of volume $V$ ). For further discussion, we set $k_{B}, \varepsilon, \sigma$ and $m$ to unity. This fixes the unit of time $\tau=\sqrt{m \sigma^{2} / \varepsilon}$ to unity as well.

We have used molecular dynamics (MD) [26, 27] simulations to study coarsening phenomena in this model. All our simulations were done in the NVT ensemble. Unless otherwise mentioned we have used Nosé-Hoover thermostat (NHT) 27, 28 for the control of temperature. In a recent paper [21] we have demonstrated that NHT serves good purpose of studying hydrodynamic phenomena in this system. In the Verlet velocity algorithm [27], that we have employed to solve the dynamical equations, the time step $\Delta t$ for integration was set to $\Delta t=0.005 \tau$. All 
of our results were obtained by applying periodic boundary conditions and averaging over multiple initial configurations.

Before quenching, homogeneous initial configurations were prepared at desired densities, via MD runs at temperature far above $T_{c}$. These homogeneous systems were quenched to $T=0.6$. As we will see, the coexistence vapor and liquid densities at this temperature are $\rho_{v}^{\mathrm{eq}} \simeq 0.007$ and $\rho_{\rho}^{\mathrm{eq}} \simeq 0.8$, respectively, giving a value of the coexistence diameter $\rho_{d} \simeq 0.4$. We obtained results by varying $\rho$ between $\rho_{v}^{\mathrm{eq}}$ and $\rho_{d}$.

For the convenience of calculation of various observables we have mapped the original continuum configurations to lattice systems via the following procedure [20, 21]. From their original positions, particles were moved to the nearest sites of a simple cubic lattice of regular spacing $\sigma$. Following this, a filled lattice site $i$ was assigned a spin value $S_{i}=+1$ and for an empty site $S_{i}=-1$. Further, to deal with pure domain morphology, we have eliminated the thermal noise, over length scale $\xi$, via a majority spin rule [10, 11]. The analysis with this system thus becomes analogous to low-temperature Ising model.

From these mapped configurations, the two-point equaltime correlation function, $C(r, t)$, was calculated as [2]

$$
C(r, t)=\left\langle S_{i} S_{j}\right\rangle-\left\langle S_{i}\right\rangle\left\langle S_{j}\right\rangle, \quad r=|\vec{i}-\vec{j}| .
$$

In the rest of the paper we will deal with $C(r, t)$ normalized to unity at $r=0$. The average domain size $\ell$ was obtained from the decay of $C(r, t)$ as

$$
C(r=\ell, t)=h,
$$

by setting $h=0.25$. Note that for self-similar pattern dynamics, $C(r, t)$ exhibits the scaling form

$$
C(r, t) \equiv \tilde{C}(r / \ell(t)),
$$

where $\tilde{C}(\tilde{x}, t)$ is a master function independent of time. We have also calculated the domain size distribution function $P\left(\ell_{d}, t\right)$ where $\ell_{d}$ is the distance between two successive domain boundaries in $x-, y-$ or $z$-directions. From normalized $P\left(\ell_{d}, t\right), \ell$ was estimated as

$$
\ell=\int d \ell_{d} \ell_{d} P\left(\ell_{d}, t\right)
$$

We have checked that the results obtained from Eqs. (15) and (17) are same, apart from a proportionality factor. So, in the rest of the paper we will stick to $\ell$ from Eq. (15) only. Here we mention that $P\left(\ell_{d}, t\right)$ exhibits the scaling form [2]

$$
\ell(t) P\left(\ell_{d}\right) \equiv \tilde{P}\left(\ell_{d} / \ell(t)\right)
$$

where $\tilde{P}(\tilde{y})$ is a time independent function.

In addition to the calculations discussed above, we have estimated various other quantities. Also important methodologies of analysis were used. These we will discuss in the next section while presenting the results.

\section{RESULTS}

We begin with the presentation of evolution snapshots for an off-critical density $\rho=0.08$, in Fig. 1 Pictures are

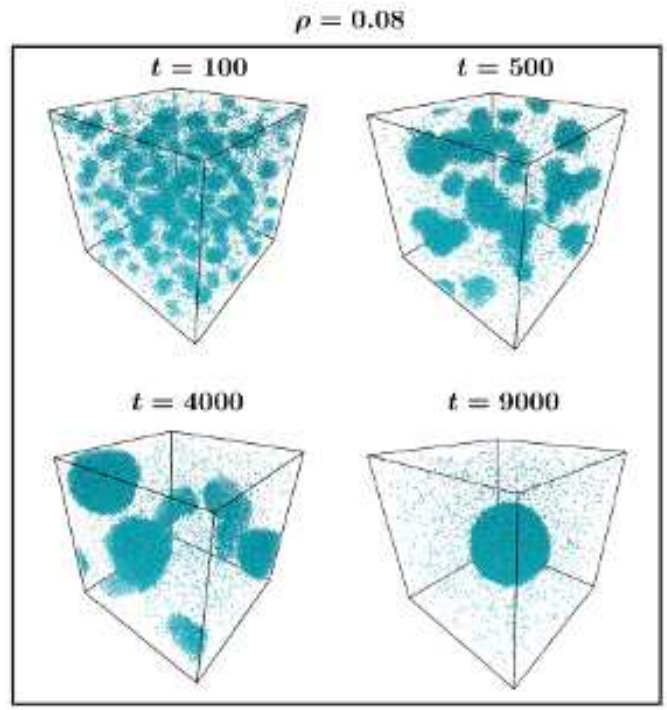

FIG. 1. Evolution snapshots after quenching a homogeneous Lennard-Jones system, of density $\rho=0.08$, to $T=0.6$. The dots mark particle positions. Periodic boundary conditions were applied in all directions. The linear dimension of the cubic box is $L=80$.

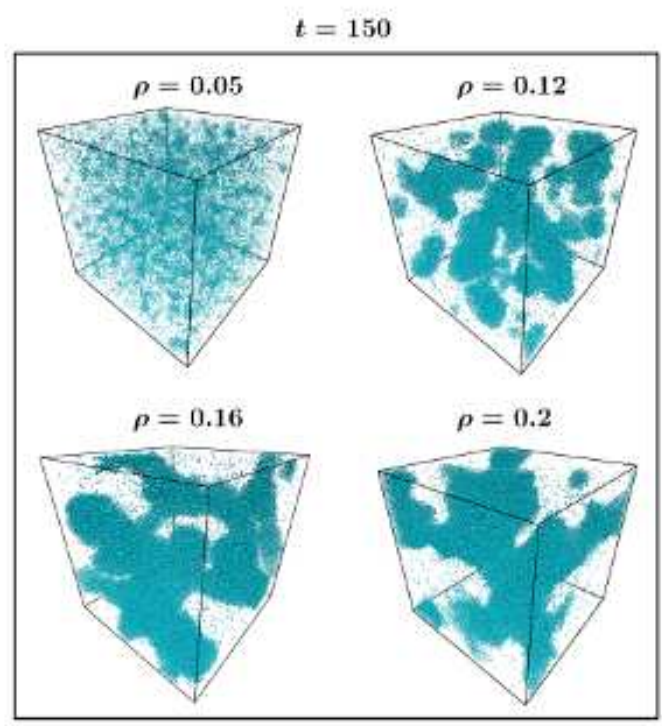

FIG. 2. Snapshots during the vapor-liquid phase separation in the Lennard-Jones model with different overall densities. All results correspond to $t=150$ and $L=80$.

shown from four different times demonstrating the formation and growth of spherical liquid droplets in vapor background. Note that the dots in these snapshots represent particle locations. Here we point out that, due to fluctuations, the droplets have spherical symmetry only in average statistical sense.

For an understanding of the density dependence of the domain pattern, in Fig. 2 we show snapshots from four different densities, all from the same time. While for the lowest value of $\rho$ domains have just begun to form, for large enough $\rho$, the domains are quite robust. This indicates that the nucleation gets delayed with the approach 
towards the coexistence curve.

From the snapshots of Fig. 2 it is clear that the densities, at least, upto $\rho=0.12$ are low enough so that the interconnected liquid domains are not stable. On the other hand, for $\rho \geq 0.16$, percolating domain structure is clearly visible. One may ask the question here, if the spinodal line should pass through a density between these values, at this temperature. Our primary objective in this paper, however, is to estimate the difference in domain growth mechanisms between the droplet and percolating morphologies. From Fig. 2 we have identified a reasonable boundary between the two cases. Before moving onto the results and discussions for domain growth laws in the two cases, below we identify the coexistence densities at this temperature and check, based on the overall system density, during nonequilibrium evolution how fast the density in domains reaches those equilibrium values. In addition, we will also discuss results for few morphology characterizing functions.

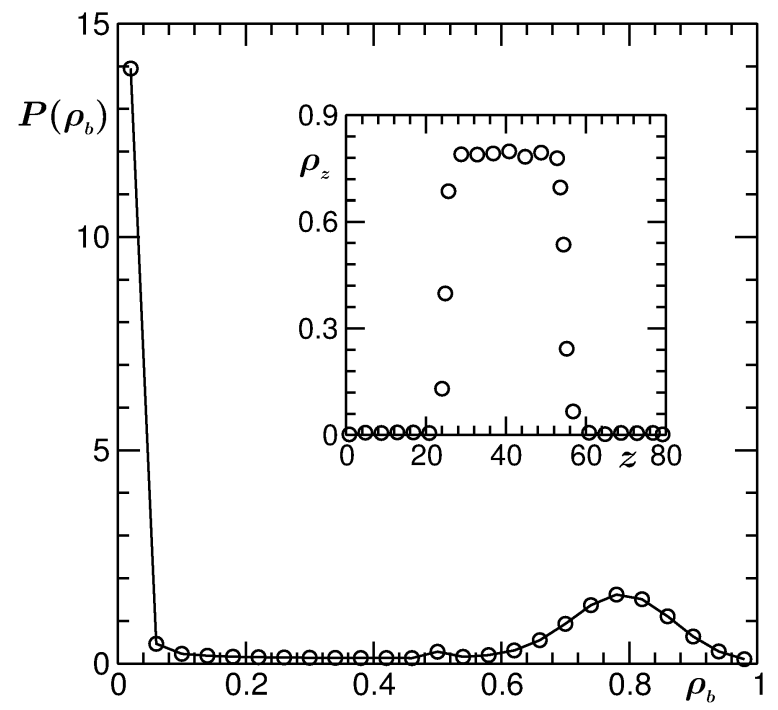

FIG. 3. Plot of the probability distribution of density, $\rho_{b}$, at different points in the system, for $\rho=0.3$ at $t=400$, with $L=64$. Note that the system is still far from equilibrium. Inset: Equilibrium density profile as a function of the $z-$ coordinate of an elongated box of $x-, y-$ and $z$ - dimensions $L_{x}=10, L_{y}=10$ and $L_{z}=80$, respectively. Again we have $\rho=0.3$. Note that apart from this one, all other results in the paper are from cubic boxes. The results are presented after averaging over 10 initial configurations.

In the inset of Fig. 3 we show the equilibrium density profile $\rho_{z}$ as a function of $z$ coordinate of a box, elongated in this direction. Because of the shape of the box, from the energy minimization condition, it is obvious that in equilibrium the interface between the vapor and liquid phases will always be perpendicular to the $z$ - direction. From the flat regions of the plot we identify that $\rho_{\ell}^{\mathrm{eq}} \simeq 0.8$ and $\rho_{v}^{\mathrm{eq}} \simeq 0.007$.

In the main frame of Fig. 3 we show the distribution of local density, $\rho_{b}$, obtained by constructing small boxes around a point including only its nearest neighbours. Note that this plot corresponds to nonequilibrium situation as opposed to the inset. Clearly two-peak structure is visible (due to small value of vapor-phase density, a maximum cannot be seen in the vapor side - for that one needs very small bin size which in turn will make the data very noisy). The nonzero width of these peaks are due to density fluctuations within the domains as well as because of contributions coming from the interfacial regions. One can, of course, obtain the average densities within the domains from the first moment of this distribution using data around each of the peaks. But the choice of the regions around the peaks is not completely unambiguous and also, the contribution from the interfacial regions cannot be separated out. So, we identify the location of the maximum as the average density (time dependent) within domains - this is reasonable considering the near symmetric look of the distribution, say, for the liquid part in Fig. 3. Note that both the main frame and the inset in this figure correspond to $\rho=0.3$. In the main part, the location of the liquid maximum is at 0.8 . This is consistent with the information obtained from the equilibrium picture in the inset which is due to the fact that the time chosen in the nonequilibrium case is rather late with respect to the pace of coarsening for this density - essentially by this time the domain order-parameter has equilibrated. But at early enough time there will be disagreement as seen below.

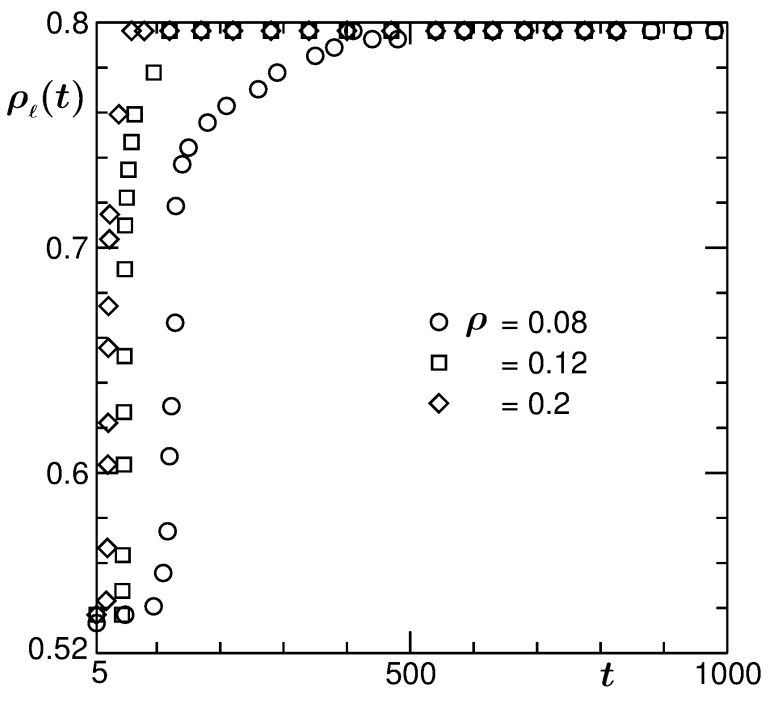

FIG. 4. Plots of the average density, $\rho_{\ell}$, inside liquid domains vs $t$, for different values of $\rho$. All results correspond to an averaging over 10 independent initial configurations with $L=$ 80.

In Fig. 4we present the liquid domain order parameter, $\rho_{\ell}(t)$, as a function of time for different overall densities. It is clearly noticeable that $\rho_{\ell}$ equilibrates faster with increasing supersaturation. Combining informations from Figs. 2 and 4, we conclude that the time scales of equilibration within domain and that within system are smaller for higher densities. This, of course, is expected and will have consequence in the growth of $\ell$ at early time for small values of $\rho$.

In Fig. 5 we show the scaled plot of the domain-size distribution, on a log-linear scale, for $\rho=0.08$, using data from three different times. Reasonable collapse of data implies self-similarity of the domain structures at different times. The solid line in this plot corresponds to an 


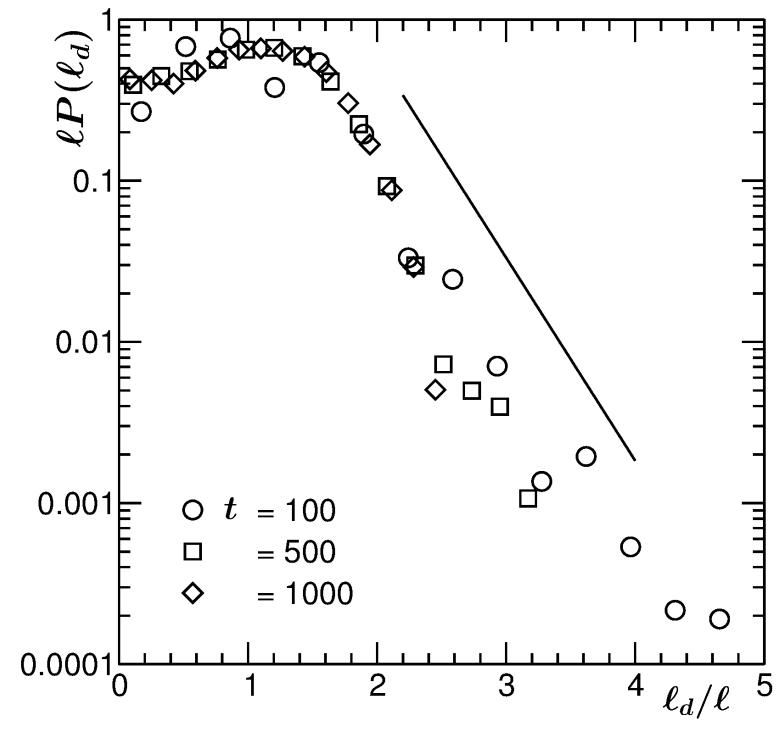

FIG. 5. Log-linear plot of the master function, $\tilde{P}\left(\ell_{d}\right)$, vs the scaled ordinate $\ell_{d} / \ell$, for $\rho=0.08$. Data from three different times are shown. The solid line represents an exponential decay. The results correspond to $L=80$ and averaging over 10 independent initial configurations.

exponential behavior. In the large domain-size limit $\tilde{P}$ is reasonably consistent with this [29, 30]. Flatter look of the data, for small abscissa variable, from later time is due to the fact that at very early time when the droplets are in the process of being nucleated, they have nearly uniform size and with the progress of time, the size dispersion increases giving rise to a larger width of the distribution.

It will be interesting to compare the scaling function $\tilde{P}$ for various densities. In Fig. 6(a) we present results from three values of $\rho$. In all the cases exponential decay of the tails is visible, though they have different slopes on the log-linear plot 29]. In Fig. 6(b) we plot this slope $m$ as a function of $\rho$. It appears that $m$ sharply falls upto the percolation density and remains constant beyond that. In the droplet regime, this fall may be expected because, with increasing supersaturation larger droplets become available, implying larger dispersion.

Exercises similar to Figs. 5 and 6 are done in Fig. 7 but this time with the correlation functions. In Fig. 7(a) we show the plots of $C(r, t)$ as a function of the scaled distance $r / \ell(t)$ for $\rho=0.08$. Data from three different times are used, as indicated. Nice collapse of data again indicates self-similarity of patterns at different times. A comparative picture of this scaling function for different densities is demonstrated in Fig. 7(b). It is observed that the depth of the minimum increases with increasing density [29].

Next, in Fig. 8 we show the plots of $\ell$ vs $t$ for few different densities. As is directly visible in Fig. 2, here also it is clearly seen that the growth occurs faster for higher density. Furthermore, there is a sudden jump in the growth rate between densities 0.12 and 0.16 . As seen in Fig. 2, between these two values the morphological change from droplet to interconnected structure occurs. The consistency of the data set for $\rho=0.2$, over reason-
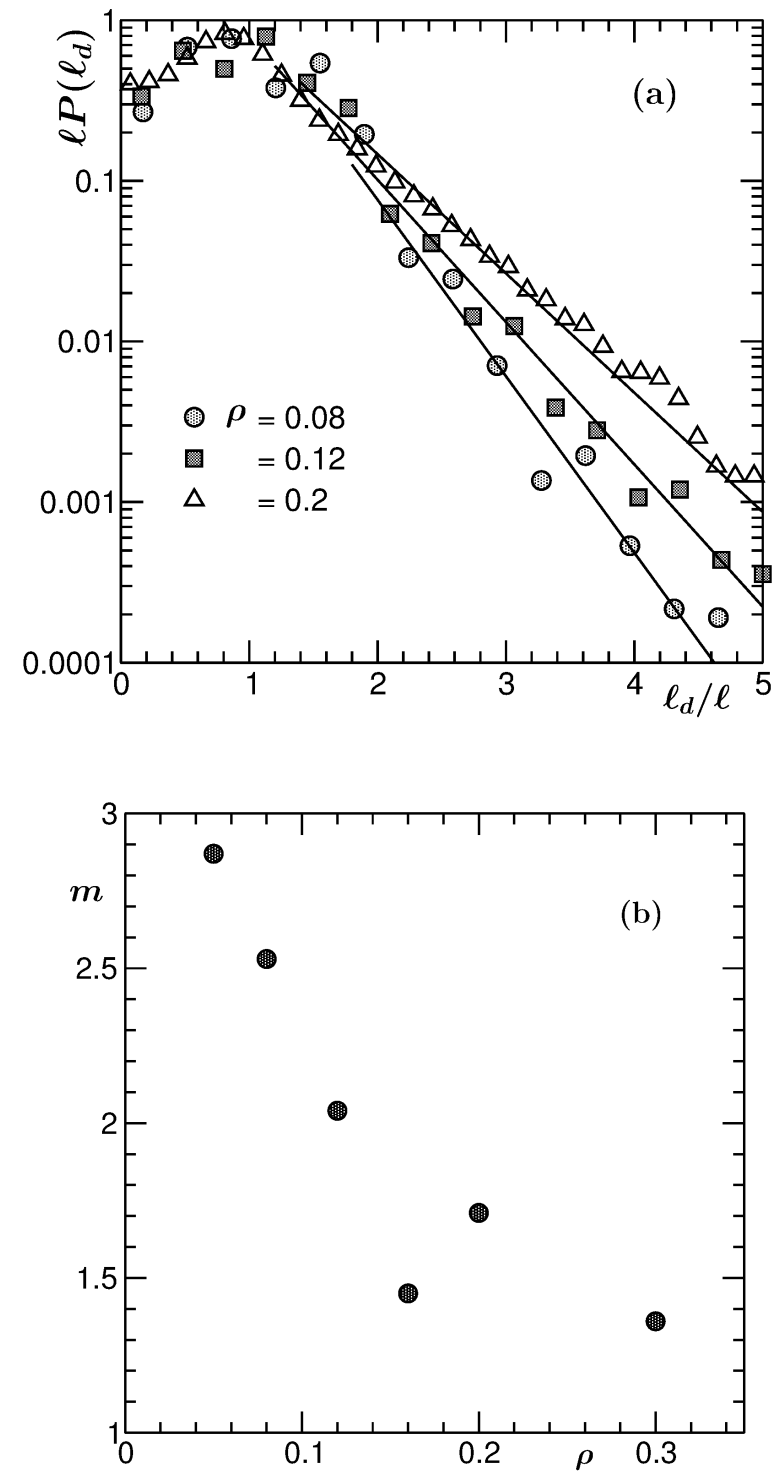

FIG. 6. (a) Plots of the master domain-size distribution functions, $\tilde{P}\left(\ell_{d}\right)$, vs $\ell_{d} / \ell$ on log-linear scale, for three different values of density, at $t=100$. The solid lines correspond to fits to the tails of different data sets. (b) Plot of the slope, $m$, of the straight lines in (a), vs $\rho$. The system size and statistics are same as Fig. 5 .

able ranges of time and length, with the solid straight line is indicative of the fact that well above the percolation transition growth is linear in time. The deviation from this behavior at later time is due to the finite-size effects [31]. Here we note that the linear domain growth for the critical density in this model (for a slightly higher temperature) was confirmed via various different methods of analysis recently 24,31 . A nearly linear behavior is also seen in the data for $\rho=0.16$, however, we cannot confirm the actual exponent in this case from this plot. We will take a relook at it later.

In Fig. 9(a) we show the $\ell$ vs $t$ data for $\rho=0.05$ on a double-log plot. Results from various different system sizes are included. A flat part at the beginning indicates delayed nucleation. This is followed by a fast growth with 

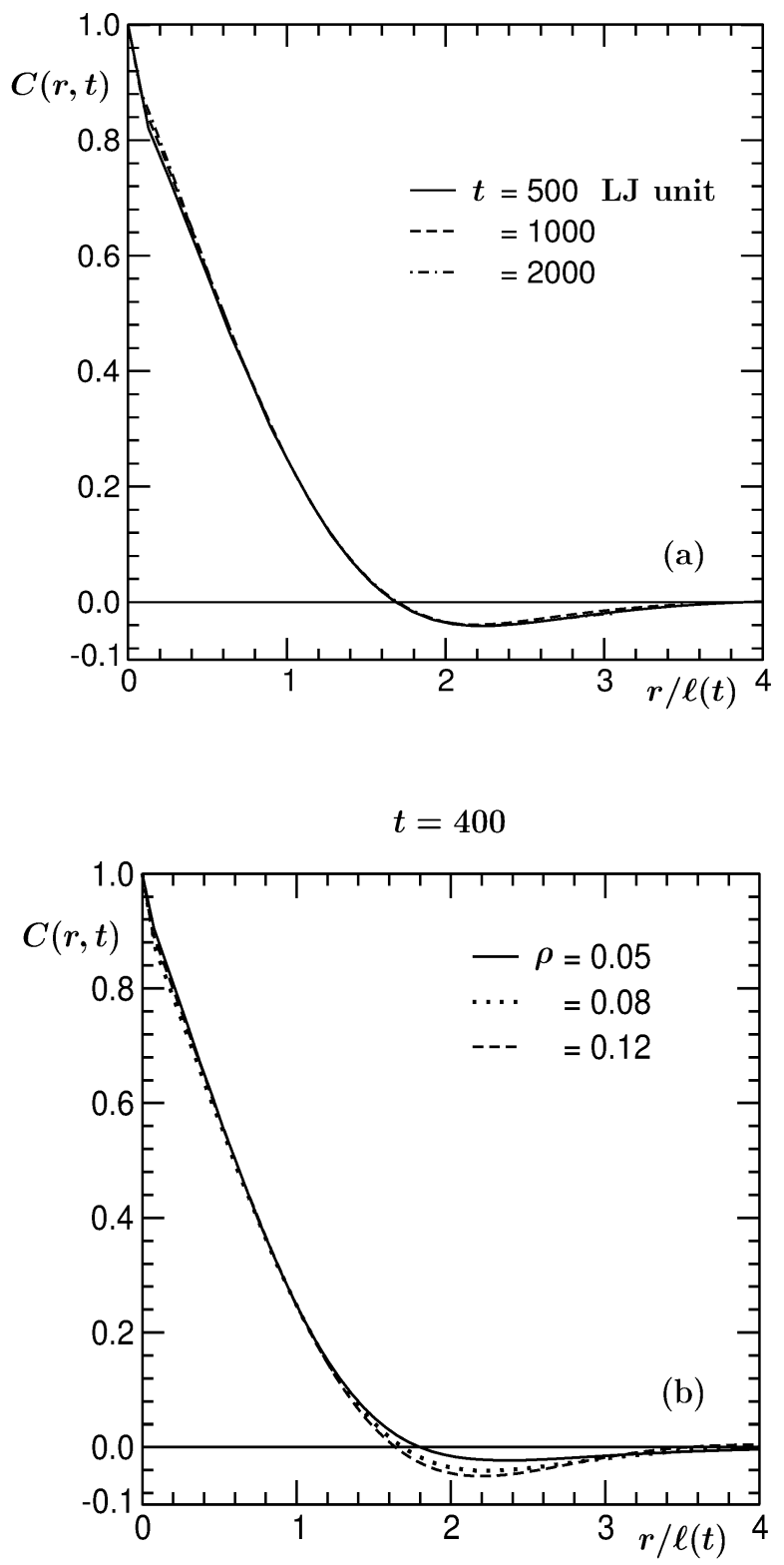

FIG. 7. (a) Plots of $C(r, t)$, vs the scaled distance, $r / \ell(t)$, for $\rho=0.08$, from three different times. The values of $\ell(t)$ was obtained from the decay of $C(r, t)$ to $1 / 4$ th its maximum value. (b) Plot of $C(r, t)$ vs $r / \ell(t)$, for different densities at time $t=400$. The results were obtained from an averaging over 10 initial configurations with minimum value of $L=80$.

exponent much higher than $1 / 3$. This can be attributed to the connectedness of unsaturated liquid domains at early time, as mentioned earlier. Finally, at very late time when there are well formed droplets the growth is consistent with an exponent $1 / 3$, over time range more than a decade. To further confirm the late time exponent value, we take the following finite-size scaling analysis 32] route. In this case, of course, the data in Fig. 9(a) clearly show an $1 / 3$ exponent. But such finite-size scaling exercise [11], as in Fig. 9(b), can prove useful in systems with strong finite-size effects. Here note that for a variety of phase separating systems, we have recently shown that the finite-size effects are rather weak [31]. Here also our

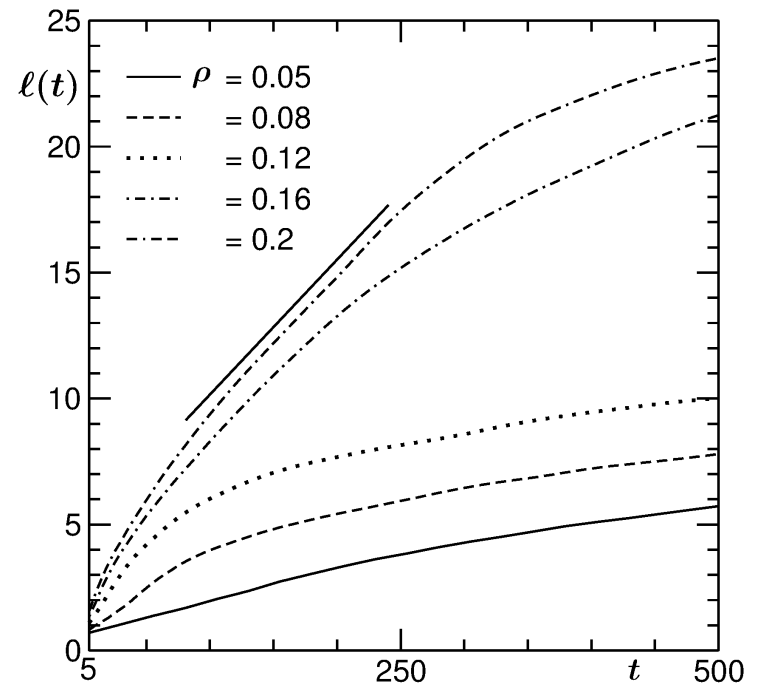

FIG. 8. Plots of the average domain size, $\ell(t)$, vs $t$, on linear scale, for different densities. The solid line corresponds to a linear behavior. All the results are presented after averaging over 10 initial configurations with lowest value of $L$ being 80 .

results appear consistent with that picture. This can be appreciated from the fact that, in Fig. 9(a), almost all the way upto equilibrium, data for a smaller system follows that of a larger one.

In the analysis in Fig. 9(b), we use the fact that the equilibration time, $t_{\text {eq }}$, for a system of size $L$ should scale with each other as

$$
L \sim t_{\text {eq }}^{\alpha}
$$

This is similar to finite-size scaling analysis of a quantity $X$ in equilibrium critical phenomena by computing it at finite-size critical temperatures and extracting the corresponding critical exponent from the plot of $X$ vs $L$. In the present case the inverse of equilibration time for a particular value of $L$ is analogous to the deviation of the finite-size critical temperature from the corresponding thermodynamic value. This latter quantity is related to $L$ via power-law with exponent $\nu$. We stress that the data in Fig. 9(b) reconfirms that the growth exponent is $1 / 3$. Similar exercises with other densities (with droplet morphology) also lead to the same value of the exponent. For the sake of brevity we do not show them here. Rather we reanalyze the same data set via another finite-size scaling method.

In equilibrium critical phenomena singularity of $X$ is quantified as

$$
X \simeq \xi^{x / \nu},
$$

where $x$ is a critical exponent. Note that $\xi$, for a finite value of $L$, can at most be of the system size. So, at criticality

$$
X \sim L^{x / \nu} .
$$

Far away from $T_{c}$, one writes 33 .

$$
X=L^{x / \nu} Y(y) ; y=\left(\frac{L}{\xi}\right)^{1 / \nu}
$$



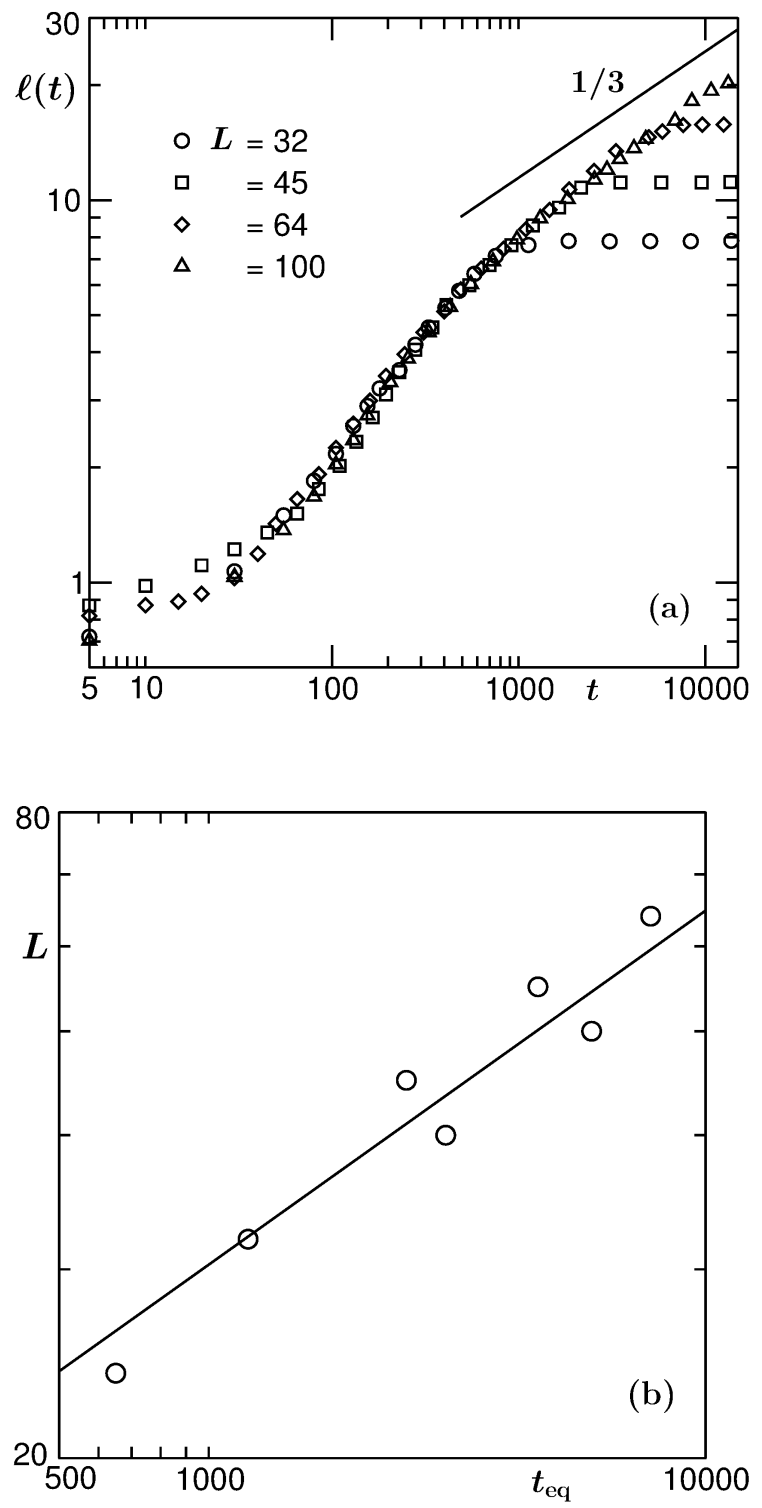

FIG. 9. (a) Log-log plots of $\ell(t)$ vs $t$ for different system sizes and $\rho=0.05$. The solid line corresponds to a power-law growth with $\alpha=1 / 3$. (b) Log-log plot of system size, $L$, vs $t_{\text {eq }}$ for $\rho=0.05$. See text for the definition of $t_{\text {eq }}$. The continuous line corresponds to $\alpha=1 / 3$. In each of the cases the statistics of averaging is at least 6 initial configurations.

where $Y$ is a scaling function independent of $L$. The behavior of $Y$ should be such that, for $T>>T_{c}$, one recovers Eq. (20) involving the thermodynamic limit value of $\xi$, thus

$$
Y(y ; y>>1) \sim y^{-x} .
$$

In a plot of $X L^{-x / \nu}$, one uses $x$ as an adjustable parameter to obtain collapse of data from different sizes. The value of $x$ that provides the best collapse is taken as the thermodynamic value of the critical exponent.

In analogy with the above discussion of finite-size scaling in critical phenomena, one can construct similar equations for the present case as [10, 11, 31, 34]

$$
\ell(t)=L Y(y)
$$
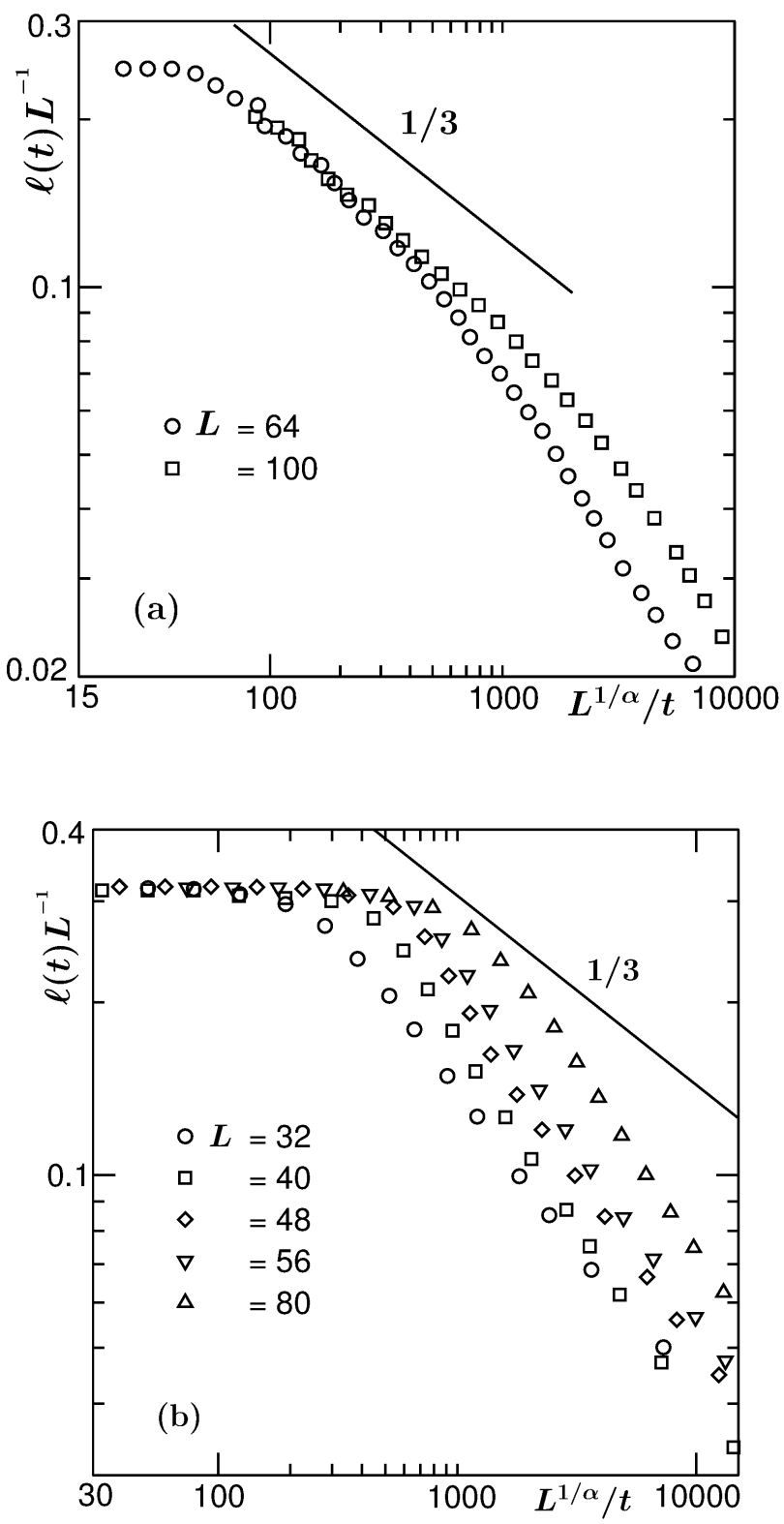

FIG. 10. (a) Log-log plot of $Y(y)$ vs $y$ for different system sizes for $\rho=0.05$, by fixing $\alpha$ to $1 / 3$. The solid line corresponds to an exponent $1 / 3$. (b) Same as (a), but for $\rho=0.16$. The statistics in (b) is slightly poorer than in (a).

$$
y=\left(\frac{L}{\ell}\right)^{1 / \alpha} \propto \frac{L^{1 / \alpha}}{t} .
$$

When $\ell / L$ is plotted vs $y$, only appropriate value of $\alpha$ will provide good collapse of data from different system sizes. The collapsed data in turn will have a behavior $\sim y^{-\alpha}$ in finite-size unaffected region. Here we emphasize that such finite-size scaling often becomes necessary to avoid less reliable methods of extracting exponents from loglog plots or data fitting exercises that may suffer from inaccuracy due to presence of non-zero offsets or strong fluctuations in the data.

In Fig. 10(a), we plot $\ell / L$ vs $L^{1 / \alpha} / t$, using two data sets of Fig. 9(a). From the behavior of data in Fig. 9(a), it is clear that there are multiple scaling regimes, as already stated. So, in this finite-size scaling exercise, we do 


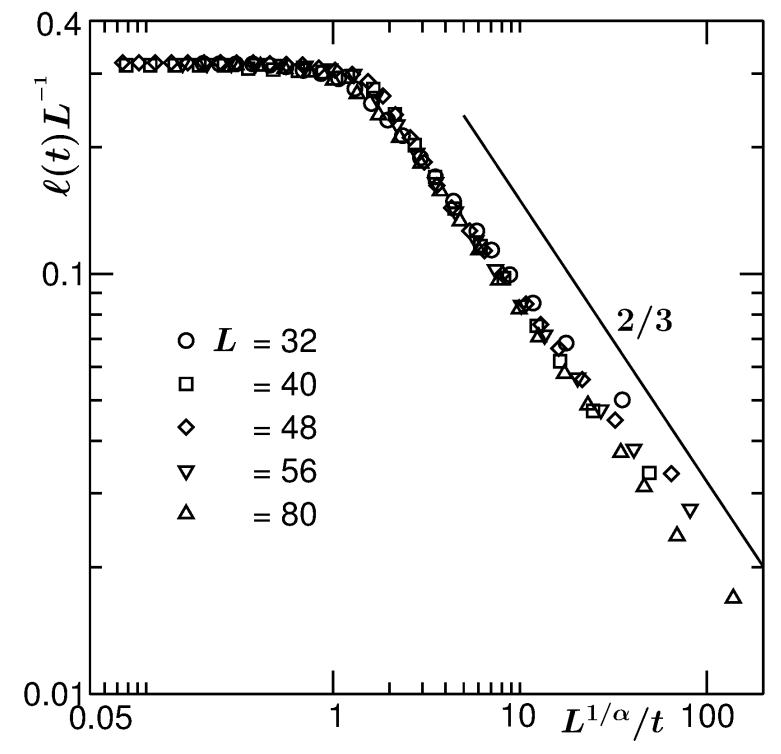

FIG. 11. Log-log plot of $Y(y)$ vs $y$ for different system sizes for $\rho=0.16$, by fixing $\alpha$ to $2 / 3$. Here the solid line represents $\alpha=2 / 3$.

not expect collapse of data in the whole range since only a single value of $\alpha$ can be used in this analysis. Since our objective for the droplet morphology is to establish the value $1 / 3$ at late time, we focus on obtaining optimum collapse in this regime only. Indeed, best collapse is achieved for $\alpha=1 / 3$ and the behavior of the master curve in the relevant region then is consistent with $y^{-1 / 3}$. In Fig. 10(b) we do the same exercise for $\rho=0.16$, again by fixing $\alpha$ to $1 / 3$. It is clear from this figure that $1 / 3$ is certainly not the right exponent for this density.

For $\rho=0.16$, desired behavior is best described for $\alpha=2 / 3$ which is shown in Fig. [11. This reconfirms that the exponents are very different for the droplet and percolating morphologies. However, a value different from $\alpha=1$, in this case, can be due to the fact that the connectedness of the morphology at this density, just above the threshold value, may not be very robust. So, the phase separation may progress via competition of growth and break-up of tubes. This, in fact, is the physical mechanism that leads to the inertial growth law. With the approach towards the coexistence diameter, these tubes become robust and the linear viscous hydrodynamic regime lives longer before crossing over to inertial regime. Nevertheless, here we only stress on the fact that the exponents in the droplet and percolating morphologies are certainly different and caution the reader that this value of $\alpha=2 / 3$ should not be taken very seriously. Note that at very early time there is a LS diffusive regime, however short it may be. In a more appropriate analysis the length and time of crossover from the diffusive regime to the hydrodynamic one need to be subtracted. However, because of the very low temperature chosen, it is difficult to identify these crossover parameters appropriately. If it becomes possible to do this analysis more accurately by incorporating this fact, one may obtain higher value of $\alpha$.

Even though, by now, we established that for droplet morphology $\alpha$ is $1 / 3$, this does not say that this value
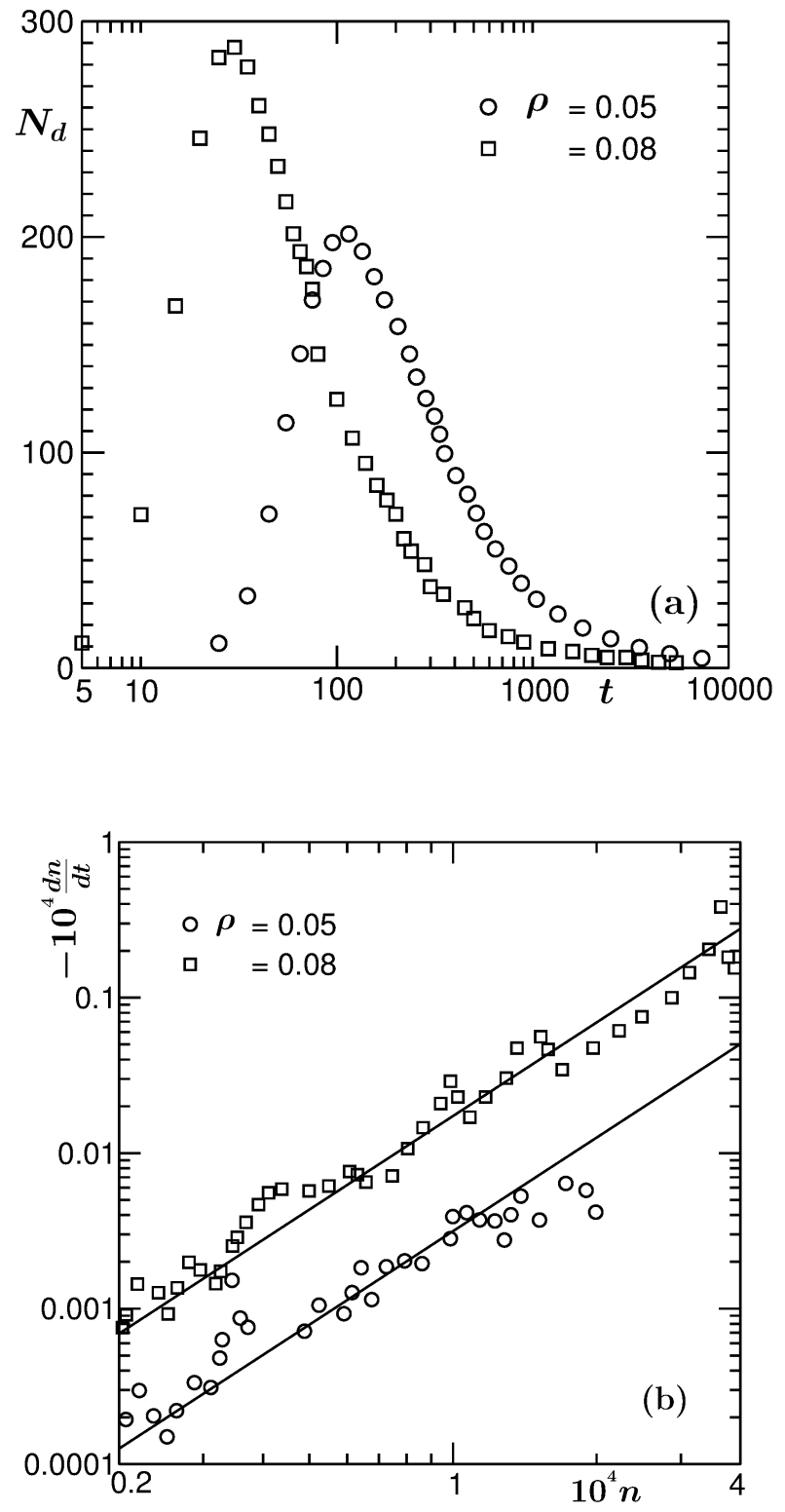

FIG. 12. (a) Linear-log plot of droplet density, $n$, in the system, vs $t$, for two different values of $\rho$, as mentioned on the figure. (b) Log-log plot of $d n / d t$ vs $n$ for the same systems as in (a). The solid lines stand for quadratic $\left(n^{2}\right)$ behavior. The ordinate for $\rho=0.08$ was multiplied by 5 .

is the result of inter-droplet collision and not due to LS mechanism. In the following we proceed to resolve that. In Fig. 12(a) we present plots of droplet density $n$ as a function of $t$ for two different values of $\rho$. For a discussion on the identification of droplets and calculation of their number, we refer the readers to Ref. [21]. This figure is quite instructive which again tells us that the nucleation gets delayed with decreasing overall density and clarifies why we have flat region in Fig. 9(a), at the beginning. The maximum in the plots is due to the fact that at early time nucleation events dominate the collision.

From the plots of Fig. 12(a), we calculate $d n / d t$ and plot it vs $n$, on double-log scale, in Fig. 12(b). The data for both the densities are nicely consistent with powerlaw carrying exponent 2 . This confirms the validity of 


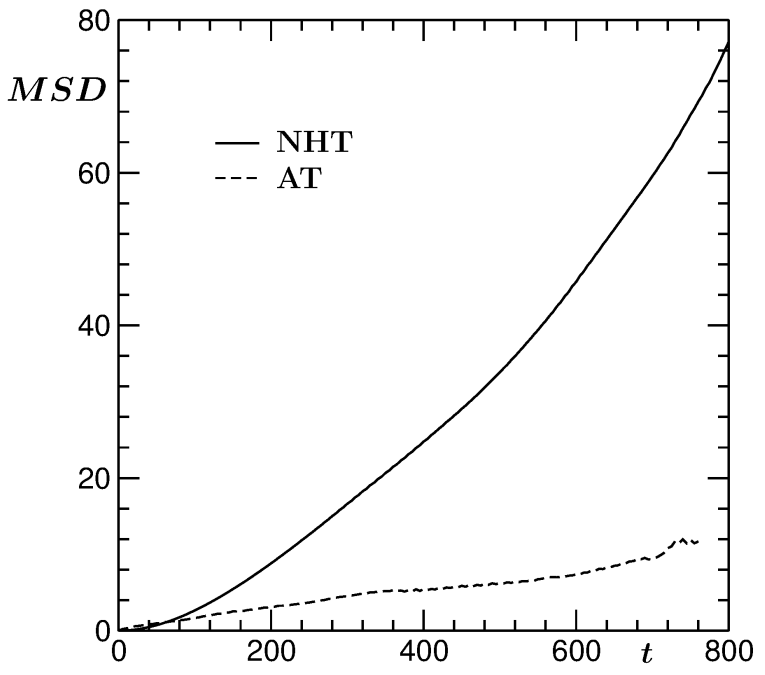

FIG. 13. Plots of the MSD of droplets of approximately same size from NHT and AT, for $\rho=0.05$. Ordinate of data for AT has been multiplied by 50 .

Eq. (2) which was written down from the assumption of droplet collisions.

Note that all our results so far were obtained by using NHT which preserves hydrodynamics well. Instead, if one applies a stochastic heat bath, e.g., Andersen thermostat (AT) 27], it is expected that the growth will be in accordance with LS mechanism. In this latter case, we do not expect fast motion of the droplets. Rather, particles from smaller droplets will get deposited on larger droplets via diffusive motion, keeping essentially the droplet centre of mass (CM) fixed. In Fig. 13 we have compared the mean squared displacements (MSD) of CMs of droplets of similar size for the NHT and AT cases. It is clearly seen that the above mentioned comparative picture is true. Note here that the AT data were multiplied by a factor $(>>1)$. This difference between the two cases confirms our claim about the droplet motion and collision mechanism further.

Supersaturation in a vapor-liquid transition can be defined as [35] $S=\rho / \rho^{\mathrm{eq}}$. Assuming that the spontaneous phase separation is related to the onset of interconnected structure, the value of $\rho$ should lie between 0.12 and 0.16 . Taking $\rho=0.14$, we obtain $S \simeq 20$. Note that from an equation of state study of a similar model, the supersaturation at the spinodal point for slightly lower temperature (as a fraction of $T_{c}$ 36]) was obtained to be 37] $S \simeq 32$. Our result can be treated consistent with that. Never- theless, we again stress that the existence of a spinodal in a system like ours is of doubtful validity [7].

\section{CONCLUSIONS}

Via molecular dynamics simulations we have studied kinetics of phase separation for vapor-liquid transition, following temperature quenches inside the coexistence region for different overall densities. It is observed that for densities closer to the vapor branch of the coexistence curve, the late time growth dynamics is due to droplet motion and collision. On the other hand, for densities above a certain crossover value, domain morphology is percolating in nature and growth occurs due to fast motion of material through elongated tube like regions. These two mechanisms give rise to significantly different exponents, for the growth kinetics [13 15, 17 19], which are estimated.

Further, we have presented important results for the functions that characterize morphology. E.g., the domain size distribution exhibits exponential tail. It is demonstrated that the decay length of this function is strongly dependent upon the overall density. Similar results are presented for the two-point equal time correlation functions. Density dependence of these quantities are discussed.

Number of interesting further studies can be done. It will be important to look at the aging property [38, 39] in this system, particularly, its dependence on the variation of density. For the characterization of morphology, in addition to the scaling functions presented here, it will also be useful to calculate the fractal dimension [40]. We plan to address these questions in future. In addition, all these studies can be repeated for fluid mixtures as well [41], even though they will be computationally very demanding, as far as molecular dynamics simulations are concerned.

\section{ACKNOWLEDGEMENT}

SKD and SR acknowledge financial support from the Department of Science and Technology, India, via Grant No SR/S2/RJN-13/2009. SR is grateful to the Council of Scientific and Industrial Research, India, for their research fellowship.

* das@jncasr.ac.in
[1] K. Binder, in Phase transformation of Materials, edited by R.W. Cahn, P. Haasen and E.J. Kramer (VCH, Weinheim, 1991), Vol.5, p.405.

[2] A.J. Bray, Adv. Phys. 51, 481 (2002).

[3] A. Onuki, Phase Transition Dynamics (Cambridge University Press, UK, 2002).

[4] R.A.L. Jones, Soft Condensed Matter (Oxford University Press, Oxford, 2008).
[5] Nucleation, edited by A.C. Zettlemoyer (Dekker, New York, 1969).

[6] F.F. Abraham, Homogeneous Nucleation Theory (Academic, New York, 1974).

[7] K. Binder, Rep. Prog. Phys. 50, 783 (1987).

[8] D. Kashchiev, Nucleation: Basic theory with Applications (Butterworth-Heinemann, Oxford, 2000).

[9] I.M. Lifshitz and V.V. Slyozov, J. Phys. Chem. Solids 19, 
35 (1961).

[10] S. Majumder and S.K. Das, Phys. Rev. E 81, 050102 (2010).

[11] S. Majumder and S.K. Das, Phys. Rev. E 84, 021110 (2011).

[12] S. Majumder and S.K. Das, in communication (2013).

[13] K. Binder and D. Stauffer, Phys. Rev. Lett. 33, 1006 (1974).

[14] K. Binder, Phys. Rev. B 15, 4425 (1977).

[15] E.D. Siggia, Phys. Rev. A 20, 595 (1979).

[16] J.-P. Hansen and I.R. McDonald, Theory of Simple Liquids (Academic Press, London, 2008).

[17] H. Tanaka, J. Chem. Phys. 105, 10099 (1996).

[18] H. Tanaka, J. Chem. Phys. 107, 3734 (1997).

[19] H. Tanaka, J. Chem. Phys. 103 (6), 2361 (1995).

[20] S. Roy and S.K. Das, Phys. Rev. E. 65, 26141 (2002).

[21] S. Roy and S.K. Das, Soft Matter, 9, 4178 - 4187 (2013).

[22] H. Furukawa, Phys. Rev. A 36, 2288 (1987).

[23] V. Privman, P.C. Hohenberg and A. Aharony, in Phase Transitions and Critical Phenomena, edited by C. Domb and J.L. Lebowitz, vol. 14, Chap. 1, Academic Press, New York, 1991.

[24] S. majumder and S.K. Das, Europhys. Lett. 95, 46002 (2011).

[25] S. Majumder and S.K. Das, to be published.

[26] M.P. Allen and D.J. Tildesley, Computer Simulations of Liquids, Clavendon, Oxford, 1987.

[27] D. Frenkel, B. Smit, Understanding Molecular Simulations: From Algorithm to Applications (Academic Press, San Diego, 2002).
[28] W.G. Hoover, in Studies in Modern Thermodynamics, Elsevier, vol 11, 1991.

[29] S.K. Das and S. Puri, Phys. Rev. E 65, 026141 (2002).

[30] A. Sicilia,Y. Sarrazin, J.J. Arenzon, A.J. Bray and L.F. Cugliandolo, Phys. Rev. E 80, 031121 (2009).

[31] S.K. Das, S. Roy, S. Majumder and S. Ahmad, Europhys. Lett. 97, 66006 (2012).

[32] M. E. Fisher, in Critical Phenomena, ed. M.S. Green, Academic Press, London, 1971.

[33] D.P. Laudau and K. Binder, A Guide to Monte Carlo Simulations in Statistical Physics, Cambridge University Press, Cambridge, 2009.

[34] D.W. Heermann, L. Yixue and K. Binder, Physica A 230 , 132 (1996).

[35] J. Wedekind, G. Chkonia, J. Wolk, R. Strey and D. Reguera, J. Chem. Phys. 131, 114506 (2009).

[36] V. G. Baidakov, S.P. protsenko, Z.R. Kozlova and G.G. Chernykh, J. Chem. Phys. 126, 214505 (2007).

[37] J.K. Johnson, J.A. Zollweg and K.E. Gubbins, Molecular Physics 78, 591 (1993).

[38] D.S. Fisher and D.A. Huse, Phys. Rev. B 38, 373 91989).

[39] M. Zannetti, in Kinetics of Phase Transitions (CRC Press, Boca Raton, 2009), edited by S. Puri and V. Wadhawan.

[40] S. H. Strogatz, Nonlinear Dynamics and Chaos: With Applications to Physics, Biology,Chemistry and Engineering, Addison-Wesley, 1994.

[41] A.G. lamorgese and R. Mauri, Physics of Fluids 17, 034107 (2005). 